\title{
Design and testing of a perimeter of increment threshold by projection
}

\section{Diseño y testeo de un campímetro de umbral incremental por proyección}

\author{
M.C. García-Domene ${ }^{1,2, S^{*}}$, M.J. Luque ${ }^{1, S}$, D. de Fez ${ }^{3, S}$ \\ 1. Universidad de Valencia. Burjassot (Valencia), Spain \\ 2. FISABIO-Oftalmología Médica. Valencia, Spain \\ 3. Universidad de Alicante. San Vicente del Raspeig (Alicante). Spain \\ ${ }^{(*)}$ E-mail: m.carmen.garcia-domene@uv.es \\ S: SEDOPTICA member
}

DOI: DOI: 10.7149/OPA.50.1.49504

\begin{abstract}
:
In the present study, we have designed and tested a perimeter for the detection of damage in the chromatic mechanisms using a video projector. To this purpose, we have characterized pixel to pixel a video projector, to account for the inhomogeneities in the projection. We have measured the tristimulus values of the projector primaries as a function of digital level, at 49 locations of the projection screen and, from them, we have arrived to a characterization model which reduces the color differences between predicted and reproduced color, in comparison with the traditional method, which generalizes the characterization of the screen center to all the screen.

With the perimeter, we measured the detection thresholds of stimuli favouring the response of the red-green and blue-yellow chromatic mechanisms of the human visual system. Stimulus size could either be constant or change with eccentricity with the size of the receptive fields of the neurons in the visual cortex. The psychophysical method of measurement used is the MOBS (Modified Binary Search).

We have studied the factors that can distort the measurements, as well as the repeatability, the specificity and the sensitivity of the device, with good results. A database has been created for the configuration of constant size stimulus. The trends shown by the data are consistent with the literature. When comparing both size configurations, the peripheral sensitivity is increased and repeatability is better with the stimulus size adapted to the eccentricity
\end{abstract}

Key words: perimeter, projector, chromatic visual field, visual mechanisms

\section{RESUMEN}

En el presente estudio se ha diseñado y testeado un campímetro de detección de anomalías en la percepción cromática utilizando un proyector de vídeo (Multicampímetro). Para ello se ha caracterizado colorimétricamente un proyector de vídeo, píxel a píxel, intentando modelizar las inhomogeneidades de la proyección. Se han determinado los valores triestímulo de los primarios del proyector, en función del nivel digital, en 49 localizaciones de la superficie de proyección y, a partir de ellas, se ha llegado a un modelo de caracterización del dispositivo que minimiza las diferencias de color entre el color predicho y el reproducido, en comparación con el método tradicional, que generaliza la caracterización del centro a toda la pantalla.

Con este perímetro, hemos medido los umbrales de detección de estímulos que favorecen la respuesta de los mecanismos cromáticos (rojo-verde y azul-amarillo) del sistema visual humano, utilizando dos configuraciones de tamaño: tamaño constante y tamaño variable con la excentricidad y adaptado a los campos receptivos del córtex visual. El método psicofísico de medida utilizado es el MOBS (Modified Binary Search). 
Se han estudiado los parámetros que pueden influir en la distorsión de la medida, así como la repetitividad, la especificidad y la sensibilidad del dispositivo, resultando favorables estos tres últimos parámetros. Se ha creado una base de datos para la configuración de estímulo a tamaño constante y se ha comprobado que los resultados de la sensibilidad de ambos mecanismos reproducen las características ya descritas en la bibliografía. Al comparar ambas configuraciones de tamaño, se encuentra mayor sensibilidad periférica y mejor repetitividad con el estímulo de tamaño adaptado a la excentricidad.

Palabras clave: campímetro, proyector, campo visual cromático, mecanismos visuales

\section{REFERENCES AND LINKS / REFERENCIAS Y ENLACES}

[1] F. Afrashi, T. Erakgun, S. Kose, K. Ardic, J. Mentes, "Blue-on-yellow perimetry versus achromatic perimetry in type 1 diabetes patients without retinopathy," Diabetes Res Clin Pract 6, 17-11 (2003). https://doi.org/10.1016/s0168-8227(03)00082-2

[2] A. Remky, O. Arend, S. Hendricks, "Short-wavelength automated perimetry and capillary density in early diabetic maculopathy," Invest Ophthalmol Vis Sci. 41, 274-281 (2000).

[3] D.R. Anderson, V.M. Patella, Automated static perimetry., St LouisMosby 1999).

[4] M.F. Delgado, N.T.A. Nguyen, T.A.Cox, et. al, "Automated Perimetry. A Report by the American Academy of Ophthalmology," Ophthalmology 109, 2362-2374 (2002). https://doi.org/10.1016/s0161-6420(02)01726-8

[5] A.J. Anderson, C.A. Johnson, M. Fingeret, et al. "Characteristics of the Normative Database for the Humphrey Matrix Perimeter," Invest Ophthalmol Vis Sci 46, 1540-1548 (2005). https://doi.org/10.1167/iovs.04-0968

[6] J.L. Keltner, C.A. Johnson, "Short-wavelength automated perimetry in neuro-ophthalmologic disorders," Arch Ophthalmol 113, 475-481 (1995). https://doi.org/10.1001/archopht.1995.01100040095033

[7] N. Fujimoto , E. Adachi-Usami, "Use of blue-on-yellow perimetry to demonstrate quadrantanopia in multiple sclerosis," Arch Ophthalmol 116, 828-829 (1998). https://doi.org/10.1001/archopht.116.6.828

[8] B.J. Lachenmayr, H. Rothbacher, M. Gleissner, Automated flicker perimetry versus quantitative static perimetry in early glaucoma. In: Heijl A, ed. Perimetry Update. Amsterdam: Kugler,359-368(1989).

[9] B. Treutwein B, “Adaptive psychophysical procedures, » Vis Res 35, 2503-2522 (1995). https://doi.org/10.1016/0042-6989(95)00016-X

[10] J.A. Phipps, A.J. Zele, T. Dang, A.J. Vingrys, « Fast psychophysical procedures for clinical testing, » Clin Exp Optom 84, 264-269 (2001). https://doi.org/10.1111/j.1444-0938.2001.tb05036.x

[11] C.A. Johnson, L.R. Shapiro, "A comparison of MOBS (Modified Binary Search) and staircase test procedures in automated perimetry. Noninvasive Assessment of the Visual System," Technical Digest Series 7, 84-87 (1989).

[12] A.J. Anderson, C.A. Johnson, “Comparison of the ASA, MOBS, and ZEST threshold methods" Vis Res 46, 2403-2411 (2006). https://doi.org/10.1016/j.visres.2006.01.018

[13] W.H. Merigan, C. Byrne, J.H.R. Maunsell, "Does primate motion perception depend on the Magnocellular pathway?” J Neurosci 11, 3422-3429 (1991).

[14] W.H. Merigan, T.A. Eskin, "Spatio-temporal vision of macaques with severe loss of $\mathrm{P}$ beta retinal ganglion cells," Vision Res 26, 1751-1761 (1986). https://doi.org/10.1016/0042-6989(86)90125-2

[15] W.H. Merigan, J.H. Maunsell, "Macaque vision after Magnocellular lateral geniculate lesions," Vis Neurosci 5, 347-352 (1990). https://doi.org/10.1017/S0952523800000432

[16] W.H. Merigan, $P$ and M pathway specialization in the macaque. From Pigments to perception. Valberg A y Lee B.B. eds. New York. Plenum Press. (1991).

[17] P.H. Schiller, N.K. Logothetis, E.R. Charles, "Functions of the colour-opponent and broad-band channels of the visual system," Nature 343, 68-70 (1990). https://doi.org/10.1038/343068a0 
[18] S.H. Hendry, T. Yoshioka, "A neurochemically distinct third channel in the macaque dorsal lateral geniculate nucleus," Science 264, 75-577 (1994). https://doi.org/10.1126/science.8160015

[19] A. Yoonessi, A. Yooonessi, "Functional assessment of Magno,Parvo and Konio-Cellular pathways; current state and future clinical applications," J Ophthalmic Vis Res 6, 119-126 (2011).

[20] Y.S. DKwak, L.W. MacDonald, "Method for characterising an LCD projection display," Projection displays VII, proceedings of the Society of photo-otpical instrumentation engineers (SPIE) 4294, 110118 (2001).

[21] J.Y. Hardeberg, L. Seime, T. Skogstad, "Colorimetric characterization of projection displays using a digital colorimetric camera," Projection displays IX, proceedings of the Society of photo-otpical instrumentation engineers (SPIE), 5002, 51-61 (2003).

[22] D.R. Wyble, M.R. Rosen, "Color management of four-primary digital light processing projectors," Journal of Imaging Science and Technology 50, 17-24 (2006). https://doi.org/10.2352/J.ImagingSci.Technol.(2006)50:1(17)

[23] D.H. Brainard, Cone contrast and opponent modulation color spaces. In Kaiser and Boynton, Human Color Vision, Optical Society of America:Washington, DC. 563-557 (1996).

[24] A.M. Derrington, J. Krauskopf, P. Lennie, "Chromatic mechanisms in lateral geniculate nucleus of macaque," J Phisyol 357, 241-265 (1984). https://doi.org/10.1113/iphysiol.1984.sp015499

[25] B. Bengtsson, "A new rapid threshold algorithm for short-wavelength automated perimetry," Invest Ophthalmol Vis Sci 44, 1388-94 (2003). https://doi.org/10.1167/iovs.02-0169

[26] B. Bengtsson, A. Heijl, "Normal inter subject threshold variability and normal limits of the SITA SWAP and full threshold SWAP perimetric programs," Invest Ophthalmol Vis Sci 44, 5029-5034 (2003). https://doi.org/10.1167/iovs.02-1220

[27] J. Rovamo, V. Virsu, “An estimation and application of the human cortical magnification factor," Exp Brain Res 37, 495-510 https://doi.org/10.1007/bf00236819

[28] M. Kalloniatis, R.S. Harwerth, "Spectral sensitivity and adaptation characteristics of cone mechanisms under white-light adaptation," J Opt Soc Am A 7, 1912-28 (1990). https://doi.org/10.1364/JOSAA.7.001912

[29] R.A. Tyrrel, D.A. Owens, "A rapid technique to assess the resting states of eyes and other threshold phenomena: the modified binary search (MOBS)," Behavior Research Methods, Instruments and Computers 106, 178-181 (1988). https://doi.org/10.3758/bf03203817

[30] B. Bengtsson, J. Olsson, A. Heijl, H. Rootzen, "A new generation of algoritms for computerized threshold perimetry, SITA,” Acta Ophthalmol Vis Scand 75, 368-375 (1997). https://doi.org/10.1111/j.1600-0420.1997.tb00392.x

[31] H. Passing, W. Bablok, "A new biometrical procedure for testing the equality of measurements from two different analytical methods. Application of linear regression procedures for method comparison studies in Clinical Chemistry,Part I," Cli Chem Cli Bioche 21, 709-720 (1983). https://doi.org/10.1515/cclm.1983.21.11.709

[32] M.A. Díez-Ajenjo, P. Capilla, M.J. Luque, “Red-green vs. Blue-yellow spatio-temporal contrast sensitivity across the visual field," J Modern Opt 58, 1736-1748 (2011). https://doi.org/10.1080/09500340.2011.606374

[33] M.C. García-Domene “Diseño de un campímetro de umbral incremental de proyección” Universidad de Alicante, España (2013) http://hdl.handle.net/10045/35676

[34] Registro de la propiedad A-238-14 Diseño de un campímetro de umbral incremental de proyección (Programa de ordenador)

[35] Registro de la propiedad A-239-14 Diseño de un campímetro de umbral incremental de proyección (Base de datos) 


\section{Introducción}

Los dispositivos de proyección de vídeo han experimentado en los últimos años una mejora de las prestaciones de reproducción del color, así como una reducción de su coste. Esto les convierte en dispositivos potencialmente interesantes para su uso en la práctica clínica optométrica, aplicados a la generación de estímulos para tests psicofísicos de diagnóstico de alteraciones del sistema visual. En particular, permitirían el diseño de campímetros. En la actualidad diversos tipos de campímetros, al ser capaces de medir pérdidas funcionales, han mostrado ser tan o más eficaces a la hora de detectar una patología que métodos basados en la detección de signos anatómicos [1,2].

El objetivo de este trabajo sería estudiar si el uso de los proyectores de vídeo como sistema de generación de estímulos en la práctica clínica es posible, centrándonos en la aplicación concreta de estos dispositivos como generadores de estímulos para campimetrías de umbral incremental [3-5]. Sin embargo, antes es necesario determinar si tienen las prestaciones para permitir la medida de umbrales con la precisión necesaria. En nuestro caso, los estímulos utilizados están modulados a lo largo de las direcciones cardinales del espacio de color. La elección de este tipo de estímulos está motivada por el hecho de que, aunque en muchos casos no es posible asegurar que una determinada patología afecta más a un mecanismo del sistema visual que otro, sí se ha puesto de manifiesto que las pérdidas funcionales ligadas a pérdidas anatómicas se detectan antes en unos canales que en otros [6-8]. La presencia, entonces, de pérdidas selectivas de sensibilidad en determinadas direcciones del espacio, constituye un potente indicador en tareas de detección de patologías. Si el método psicofísico utilizado en las medidas es lo suficientemente rápido y fiable como para que el tiempo necesario para la realización del test no sea prohibitivo en la práctica clínica [9-12], se puede obtener la máxima información caracterizando no un único mecanismo, sino comparando el estado de deterioro relativo de dos mecanismos particulares (el rojo-verde (RG) de origen parvocelular y el azul-amarillo (BY) de origen koniocelular) [13-19].

\section{Desarrollo teórico}

El estudio se articulará en dos fases. En la primera, una vez elegido un dispositivo de proyección que cumpla de partida unos requisitos mínimos de calidad, se procederá a su caracterización colorimétrica, en principio usando métodos estándar [20-22]. A partir de la misma, se estudiará si las prestaciones del proyector permiten su uso como generador de estímulos para tests psicofísicos, en particular que involucren la medida de umbrales.

Si el resultado de la primera fase es positivo, se procederá al diseño del campímetro de umbral incremental deseado. La construcción del campímetro requiere, por una parte, desarrollar un software de control y generación de estímulos y, por otra, la obtención de una base de datos de observadores normales, agrupados por edades. Se implementará un software en entorno Matlab que permitirá la medida de umbrales cromáticos, tanto en el eje rojo-verde como en el azul-amarillo del espacio de modulaciones oponentes [23,24]. Adicionalmente, se analizará la repetibilidad del dispositivo y los factores de distorsión que pudiesen influir en la medida y se realizarán medidas con sujetos patológicos (reales o simulados).

\section{Desarrollo práctico}

\section{3.a. Caracterización colorimétrica del dispositivo}

El dispositivo consta de un proyector LGAF115 con tecnología LCoS (Liquid Crystal on Silicon), controlado mediante un ordenador AMD Athlon ${ }^{\mathrm{TM}}$ XP $2400+2.00 \mathrm{GHz}$ con $2.50 \mathrm{~Gb}$ de RAM, y una pantalla mural 3M WS180, de dimensiones $180 \times 180 \mathrm{~cm}$. Las medidas colorimétricas se realizaron mediante el espectrorradiómetro SpectraScan PR-650, colocado perpendicularmente al centro de la proyección en la pantalla y para una superficie de medida de $1^{\circ}$. Los estímulos se controlaron mediante una librería de funciones para Matlab 8.0 ${ }^{\circledR}$.

Durante el proceso de calibrado, se varió de forma sistemática los distintos parámetros de configuración del proyector (luminosidad, contraste, ganancia, tamaño, etc.), midiendo para cada condición con el espectrorradiómetro, los valores triestímulos de estímulos acromáticos, generados para 11 valores equiespaciados de nivel digital. A partir de estos resultados, se seleccionaron los valores de los parámetros que minimizasen la saturación de la luminancia con el nivel digital y nos permitiesen tener un buen rango dinámico para los tres primarios (coincidiendo con los recomendados por el fabricante). Además, se comprobó la falta de homogeneidad espacial de la proyección, después de medir la luminancia de los 
primarios y del blanco en el centro y las cuatro esquinas de la pantalla. Para introducir esta inhomogeneidad, analizar la aditividad y constancia de primarios y el cálculo del perfil ICC de cada primario (relación nivel digital-luminancia), se muestreó la proyección con una rejilla de 6x8 regiones rectangulares, más el centro (49 regiones), donde se medirían las coordenadas cromáticas y luminancia de los canales RGB y el Blanco en 11 niveles digitales. Estas medidas diferían significativamente entre todas las regiones $(\mathrm{p}<0.05)$, según el test de Friedman, por lo que corroboramos la inhomogeneidad de la proyección.
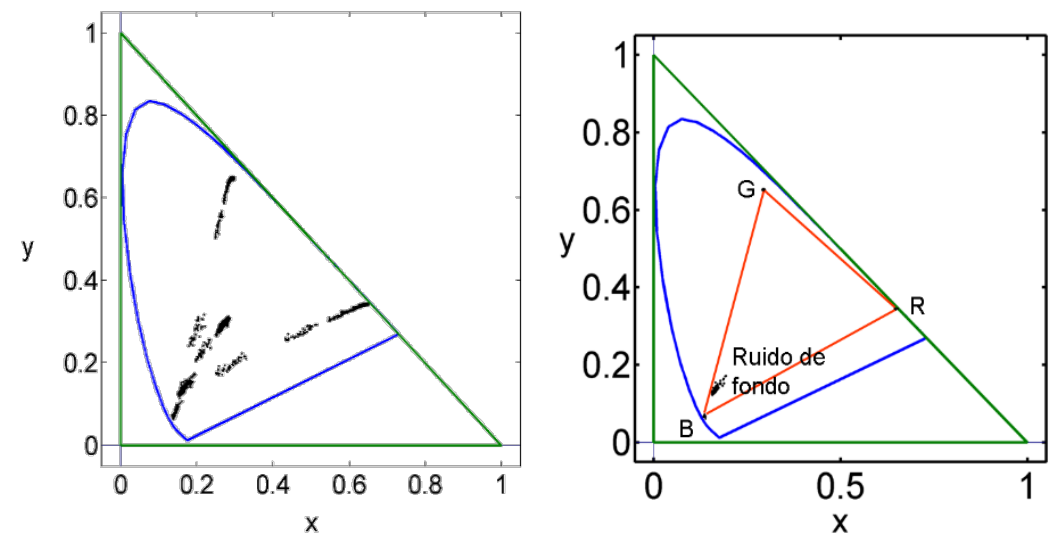

Fig. 1. Izquierda: Coordenadas cromáticas de los tres primarios y del blanco del proyector, en las 49 localizaciones espaciales de la pantalla, para los 11 niveles digitales medidos. Derecha: Estimación de las coordenadas de los primarios, asumiendo que los colores medidos corresponden a la mezcla del primario y un estímulo "ruido de fondo".

Los datos obtenidos se representan en las Figuras 1 y 2. En la Figura 1 izquierda, se muestran las coordenadas cromáticas de los primarios y del blanco del monitor, medidas para los distintos niveles digitales. El comportamiento observado puede explicarse admitiendo que, para cada nivel digital, el color resultante es la mezcla de un primario "ideal", de cromaticidad fija y luminancia dependiente del nivel digital (Figura 1, derecha) y un ruido de fondo. Esto es, para cualquier color C, los valores triestímulos [X(C), $\mathrm{Y}(\mathrm{C}), \mathrm{Z}(\mathrm{C})]$ vendrán dados por:

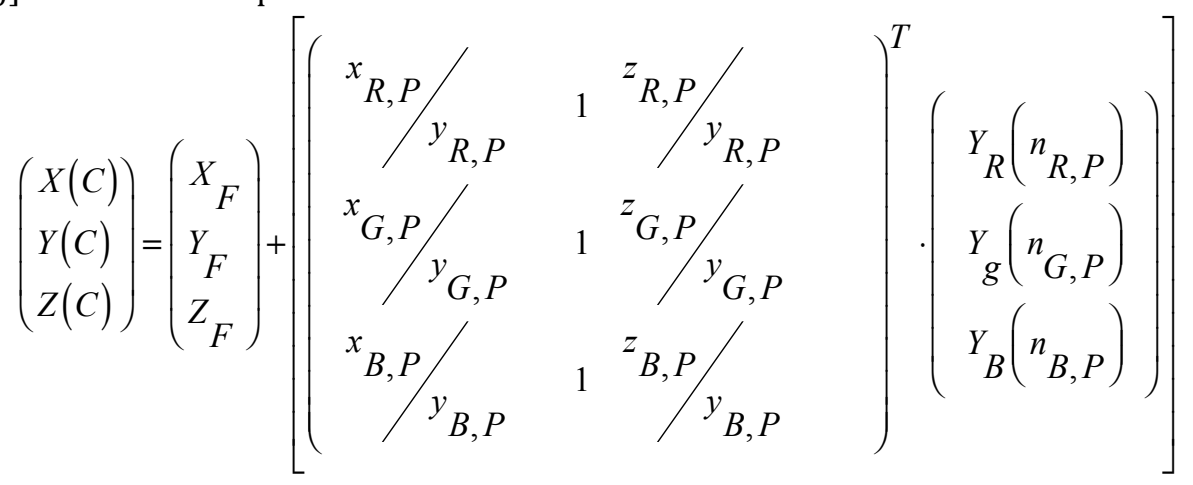

donde $\left(\mathrm{x}_{\mathrm{i}, \mathrm{P}}, \mathrm{y}_{\mathrm{i}, \mathrm{P}}, \mathrm{Z}_{\mathrm{i}, \mathrm{P})}\right.$ son las coordenadas cromáticas CIE1931 del primario i=R,G,B, en la localización $\mathrm{P}, \mathrm{Y}_{\mathrm{i}}\left(\mathrm{n}_{\mathrm{i}, \mathrm{P}}\right)$ es la luminancia del primario i para un nivel digital $n$, en la localización $P, y\left(X_{F}, Y_{F}, Z_{F}\right)$ son los valores triestímulo del ruido de fondo.

Este ruido de fondo, que se correspondería con el color generado con niveles digitales cero, no puede caracterizarse mediante el espectrocolorímetro, al ser la fiabilidad de este dispositivo baja para el nivel de lluminancias implicado. El proyector se caracteriza mediante las coordenadas cromáticas de los primarios, los valores triestímulo del ruido de fondo (Figura 1 derecha) y los parámetros de ajuste de la función luminancia vs. nivel digital, dada por:

$$
Y(n)=\frac{A \cdot n^{B}}{n^{C}+D}
$$


siendo Y(n) la luminancia, n el nivel digital y A, B, C y D los parámetros de ajuste de la sigmoide (Figura 2). Estos parámetros se han obtenido minimizando la distancia CIELab entre los valores triestímulos medidos para los primarios RGB y el blanco y la predicción de la Ec. 1.
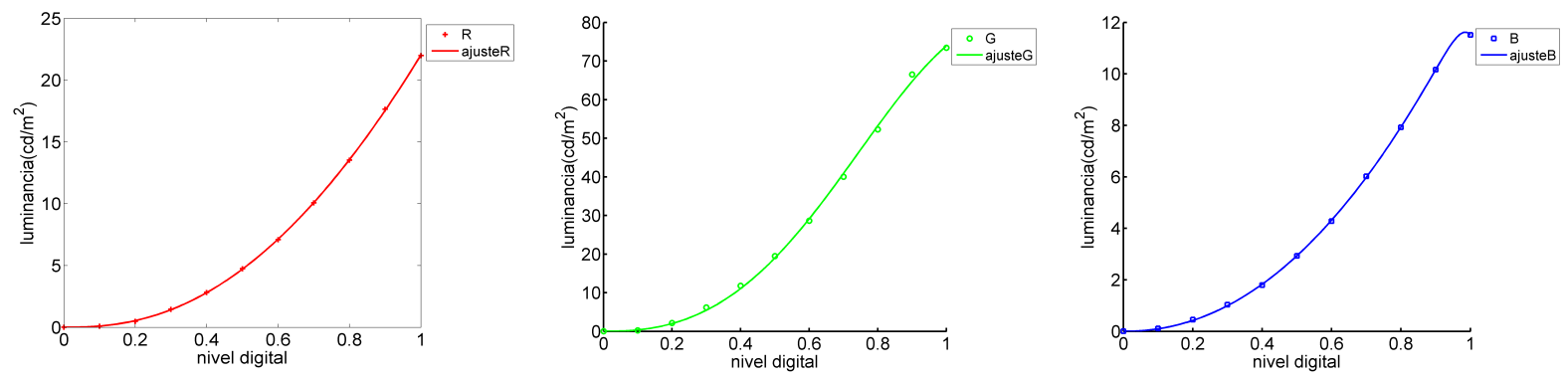

Fig. 2. Curvas luminancia frente a nivel digital para los tres primarios del proyector, obtenidas en el centro de la pantalla. Los puntos experimentales se han ajustado mediante una sigmoide.

Los parámetros de la ecuación 1 cambiaban con la localización espacial. Para caracterizar la pantalla pixel a pixel, se interpoló los parámetros obtenidos en las 49 localizaciones, usando distintos modelos. Las menores diferencias de color entre valores triestímulo experimentales y predichos por el modelo en toda la proyección se obtuvieron interpolando mediante una función superficie (función triscattinterp de Matlab).

\section{3.b. Multicampímetro}

\section{Configuración de medida}

Como podemos ver en la Figura 3 el observador se coloca a 1 metro de la pantalla, apoyando barbilla y frente en una mentonera, para evitar variaciones en la distancia de observación. En estas condiciones, el campo máximo explorable es de $56.7^{\circ}$ en vertical y $74.8^{\circ}$ en horizontal.

Exploramos un conjunto de localizaciones del campo visual con un estímulo que aparece sobre un fondo constante, que actúa como adaptador. Nuestro dispositivo permite muestrear el campo visual con dos configuraciones, una análoga a la campimetría 24-2 SWAP $[25,26]$ y otra consistente en una rejilla cuadrada 10x10 de la que se han eliminado los puntos impares y añadido el centro del campo. En ambas configuraciones, se presenta una cruz de fijación foveal, que el paciente debe mirar durante toda la medida.

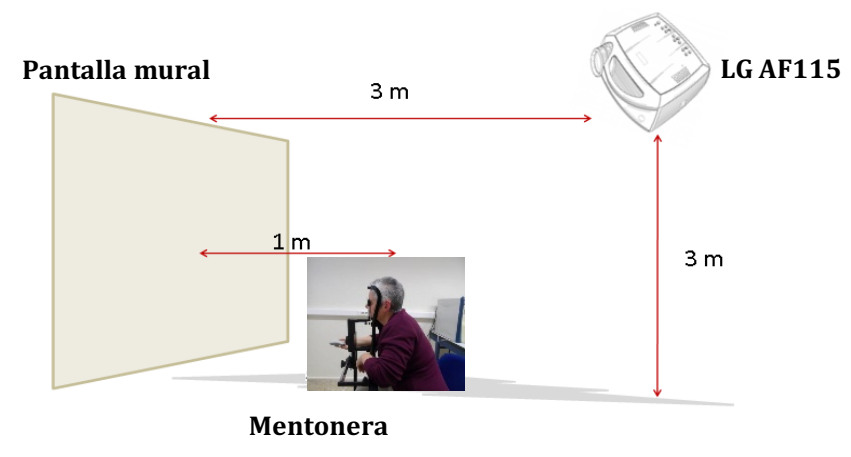

Fig. 3. Configuración de los elementos del multicampímetro.

\section{Estímulo}

Dado que queremos aislar las respuestas de los caminos visuales Parvocelular cromático y Koniocelular, los estímulos deben favorecer la respuesta de dichos mecanismos y minimizar la posibilidad de intrusión de otros mecanismos. Para minimizar la respuesta del camino Magnocelular hay que evitar los transitorios temporales al inicio y al final del estímulo, por lo que los estímulos consisten en breves secuencias de vídeo que hacen aparecer/desaparecer el estímulo progresivamente, siguiendo un perfil temporal gaussiano (Figura 4-izquierda). Para minimizar la respuesta del mecanismo acromático de origen parvocelular, debemos eliminar cualquier borde abrupto, por lo que se eligió un estímulo circular con un suavizado gaussiano del borde (Figura 4-centro). El tamaño del estímulo puede ser constante ( $1^{\circ}$ de diámetro) o modificarse en función de la excentricidad, según la función de magnificación cortical propuesta por Rovamo y Virsu [27], con lo que resultan estímulos desde $1^{\circ}$ en el centro hasta casi $10^{\circ}$ en la periferia. 

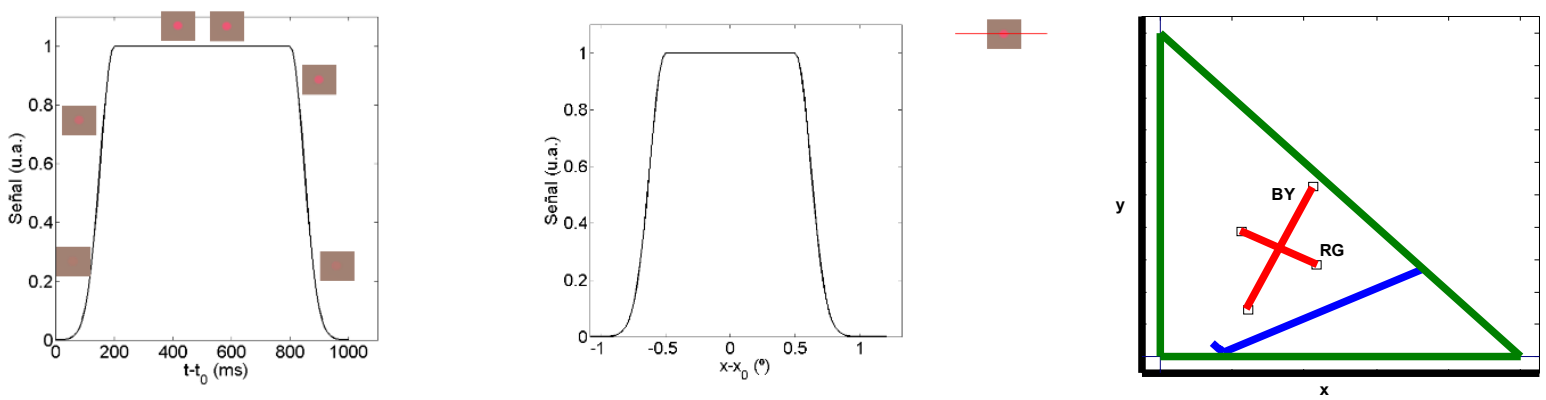

Fig. 4. Características del estímulo. Izquierda: Perfil gaussiano para presentación gradual. Centro: Perfil gaussiano para suavizar los bordes (sección horizontal). Derecha: Direcciones cardinales de la selección de colores en el espacio CIExyz.

El contenido cromático del estímulo se ha elegido para maximizar la respuesta de los mecanismos cromáticos. Para favorecer al canal Parvocelular hemos utilizado la dirección cardinal rojo-verde, RG, del espacio de modulaciones oponentes de Derrington, Krauskopf y Lennie (DKL) [23-24] y en el caso del canal Koniocelular, la dirección cardinal azul-amarillo, BY, (ver figura 4-derecha). El color del fondo, que actuará como adaptador, también se ha elegido para favorecer a los canales cromáticos. Según el estudio de Kallionatis y Harwerth [28] un fondo muy luminoso desensibiliza los canales acromáticos, pero en un proyector de vídeo disminuye la gama de colores reproducibles, así que llegamos al compromiso de seleccionar la luminancia más elevada que nos permitiera la reproducción de una gama de colores razonable: un fondo de valores triestímulo CIEXYZ [30 30 30]. Podemos ver un ejemplo de los estímulos en la figura 5. Para que todos los observadores partan del mismo estado de adaptación, se presenta durante un minuto este estímulo, antes de comenzar la campimetría.
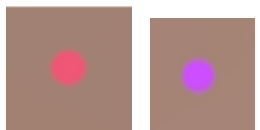

Fig. 5. Ejemplos de estímulos para tamaño constante con la excentricidad para el mecanismo RG (izquierda) y BY (derecha).

El método de medida utilizado para encontrar el umbral de sensibilidad al contraste cromático es el método MOBS de Tyrrel y Owens [29]. Este método todavía no había sido utilizado en campimetrías, pero es más rápido y preciso en test psicofísicos que otros métodos, como el SITA [30]. Fue necesario realizar algunas modificaciones para minimizar el tiempo de medida y la correcta obtención del umbral. Además de los estímulos que nos van a permitir medir el umbral, debemos asegurarnos de que el paciente realiza bien la campimetría. Para ello hemos introducido unos estímulos de control de falsos positivos, falsos negativos y pérdidas de fijación por el método de Heijl-Krakau, utilizando la localización del punto ciego del paciente determinado con nuestro software antes de comenzar la campimetría.

\section{Testeo y Resultados}

Se ha evaluado cómo influye en el resultado de la campimetría el estado refractivo del paciente, el efecto de la fatiga y del aprendizaje y la repetitividad de la medida a corto plazo. Se ha realizado una pequeña base de datos, comparando los resultados con los datos existentes en la literatura y se han medido pacientes con patologías reales o simuladas. En este estudio nos hemos centrado en la rejilla cuadrada y en el estímulo de tamaño constante.

El protocolo de medida seguido consta de una breve explicación al sujeto de la prueba que se va a realizar, seguidamente se le facilita el consentimiento informado. Una vez leído este y firmado, se realiza un examen refractivo y el test Farnsworth Munsell de 100 tonos para evaluar la presencia de alguna anomalía cromática. A continuación, se ocluye un ojo al paciente, se ubican las lentes correctoras en la mentonera si es necesario y se coloca el paciente apoyando la barbilla y la frente en la mentonera.

\subsection{Tolerancia al desenfoque.}

Un observador experimentado realizó la campimetría sin desenfoque y con $+0.75+1.5,+2$ y +3 D de desenfoque, tanto para el mecanismo RG como para el BY. El test de los rangos de Wilcoxon indica que las 
diferencias con la campimetría sin desenfoque no son significativas, por lo que podemos concluir que nuestro Multicampímetro tiene una alta tolerancia al desenfoque, al menos hasta $3 \mathrm{D}$.

\subsection{Repetitividad}

Evaluamos el efecto de la fatiga y del aprendizaje en dos observadores experimentados. Con este estudio, además, vamos a cuantificar cuántas repeticiones son necesarias para el estudio de repetitividad. Cada observador realizó 5 medidas del mismo mecanismo, intercalando descansos de 30 minutos. El resultado muestra que no existe una tendencia de variación en la sensibilidad media al realizar varias campimetrías seguidas, y tampoco existe un aumento o disminución del tiempo de realización que no sea la debida al azar. A la vista de estos resultados, pudimos concluir que dos repeticiones eran suficientes para el análisis de repetitividad de nuestro dispositivo, con un descanso de 30 minutos para evitar los efectos de la fatiga.

Para analizar la repetitividad hemos evaluado la repetibilidad mediante el test estadístico de Kruskal Wallis y la concordancia con el test gráfico de Passing-Bablock [31]. Participaron un total de 51 observadores, que realizaron 2 campimetrías, separadas por una pausa de 30 minutos, para el mecanismo RG o para el BY. Se trataba de pacientes con experiencia en tests psicofísicos, pero sin experiencia previa en este campímetro, sin patologías oculares y sin medicación que pudiera alterar la percepción o la atención del paciente. La agudeza visual con compensación era de al menos 0,5 en escala decimal. La graduación en gafa debía estar entre +5 D y -5 D, con un astigmatismo no superior a 3 D, para minimizar los errores debidos a la aberración de las lentes compensadoras.

Los resultados muestran que para ambos mecanismos las medidas son repetibles, exceptuando alguna localización periférica. En el centro del campo visual la repetitividad es buena mientras que en la periferia es menor, debido a la mayor variabilidad en las medidas producida por una inferior sensibilidad.

\subsection{Base de datos de normalidad}

Cualquier dispositivo experimental que vaya a ser utilizado para la detección de patologías o anomalías, necesita una base datos de observadores normales. Por lo que hay que comprobar si un patrón de sujetos jóvenes y sanos, determinado con este dispositivo, muestra las características básicas que, según la bibliografía, esperaríamos de las campimetrías de los mecanismos RG y BY, en particular respecto a la variación de la sensibilidad con la excentricidad y las asimetrías existentes.

Participaron un total de 55 observadores de 20 a 40 años, con los mismos criterios de inclusión que en el estudio de repetitividad, pero con un AV de al menos 0.8 en escala decimal. Los resultados, que pueden verse en la figura 6, muestran que en los campos visuales cromáticos la sensibilidad es mayor en el centro que en la periferia, con una mayor velocidad de caída de la sensibilidad con la excentricidad para el mecanismo RG que para el BY siendo, además, éste último más sensible. El hemicampo nasal es más sensible que el temporal y más sensible el inferior que el superior. Estos resultados concuerdan con los encontrados en la literatura [32].

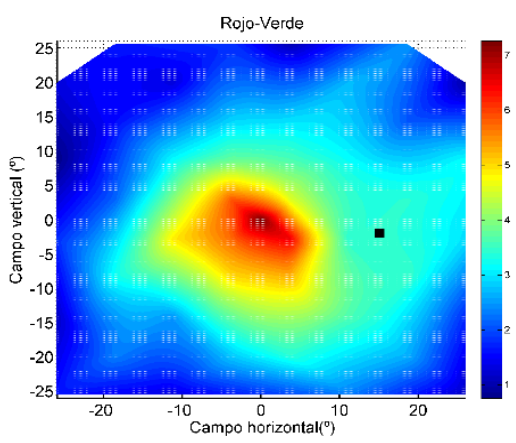

b)

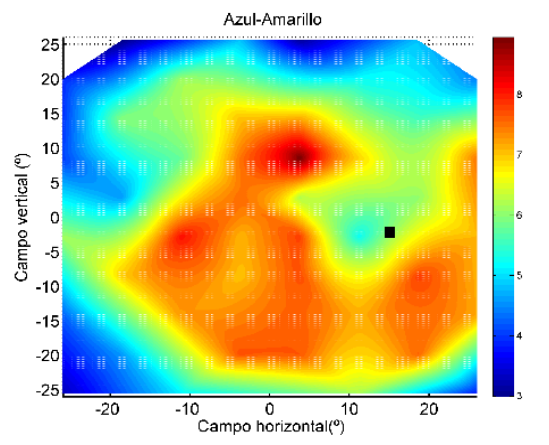

Fig. 6. Sensibilidad promedio (escala de color) para el estímulo constante con la excentricidad: a) RG y b) BY

\subsection{Aplicación a detección de anomalías}

Un test de diagnóstico debe ser capaz de identificar como sujetos sanos los que lo son (especificidad) y detectar pacientes patológicos (sensibilidad). Con este objetivo se ha realizado una serie reducida de pruebas con pacientes reales, con problemas diagnosticados y detectables por otros tipos de perímetros y que nuestro dispositivo debería detectar, si funciona correctamente. 

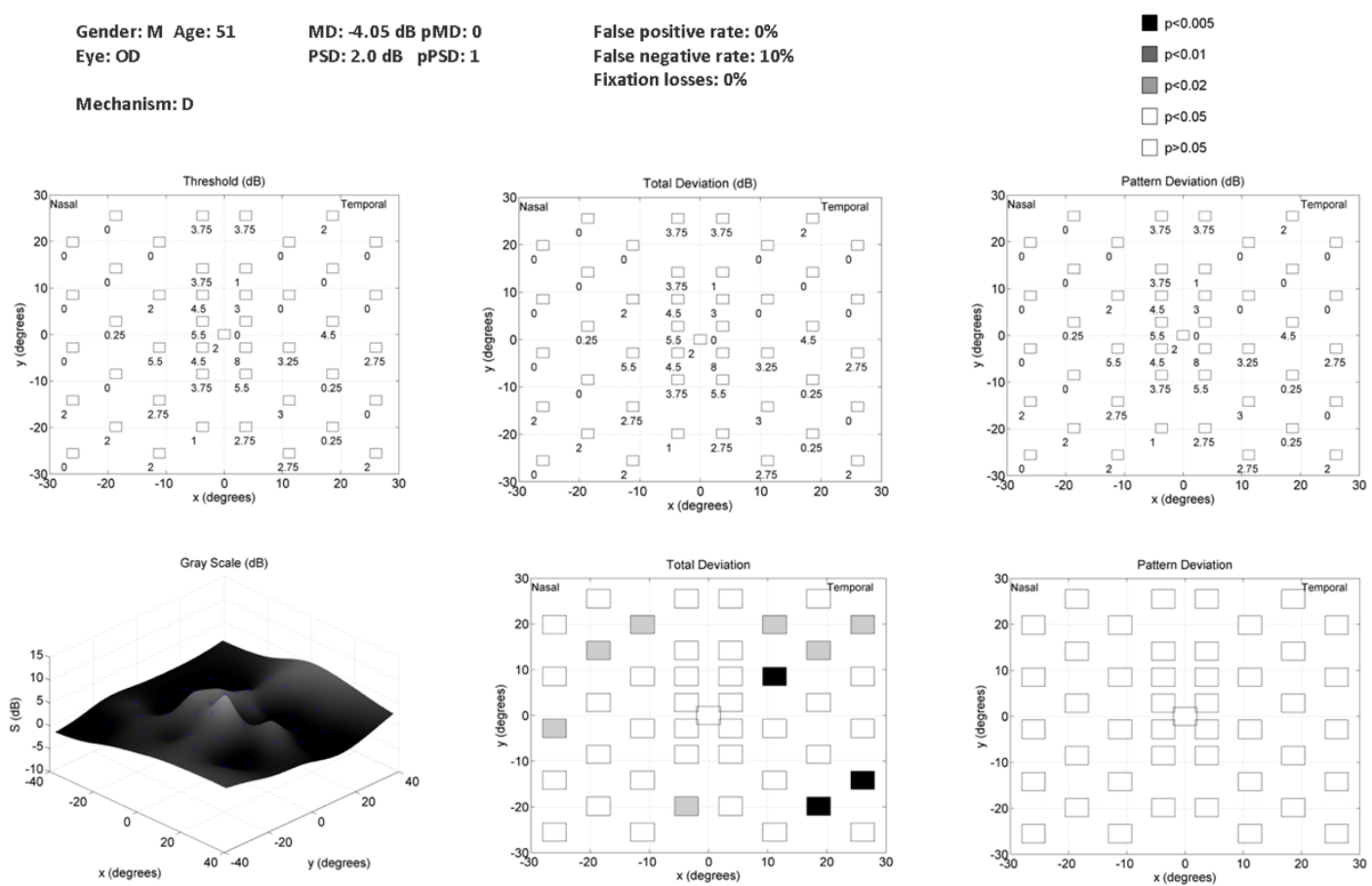

Fig. 7. Resultados para del mecanismo BY del ojo derecho, para un paciente de 51 años con cicatriz en retina.

Se realizaron medidas de un paciente con una cicatriz en retina, de una persona de 65 años para evaluar la afectación del amarilleamiento del cristalino, de un paciente joven con una deficiencia en la discriminación cromática simulada mediante filtro de color amarillo y de dos personas jóvenes sanas, Se creó una hoja de resultados, como vemos en el ejemplo de la Figura 7, donde aparece: el defecto medio y su significación, la desviación estándar del patrón y su significación, mapa de sensibilidades (2D y 3D), mapa de diferencias, mapa de probabilidad de las diferencias y mapa de diferencias de sensibilidad corregido. A partir de los resultados obtenidos de todos los sujetos, concluimos que la sensibilidad del dispositivo parece buena en el mecanismo RG, pero no está tan claro para el mecanismo BY, dado que no se muestran tantas pérdidas de sensibilidad como en el RG. Analizando los resultados de los sujetos normales, el Multicampímetro parece tener una buena especificidad, aunque más medidas serían necesarias para confirmar estos hechos.

\subsection{Estímulos adaptados a la excentricidad.}

Como habíamos previsto, los resultados periféricos para el patrón del Multicampímetro de tamaño de estímulo constante están en el límite del rango dinámico del dispositivo, lo que puede llevarnos a un mal diagnóstico de una patología. Por eso hemos realizado un pequeño estudio con el Multicampímetro de estímulo adaptado a la excentricidad. Diez sujetos sanos jóvenes realizaron la medida para el RG y otros diez para el BY.
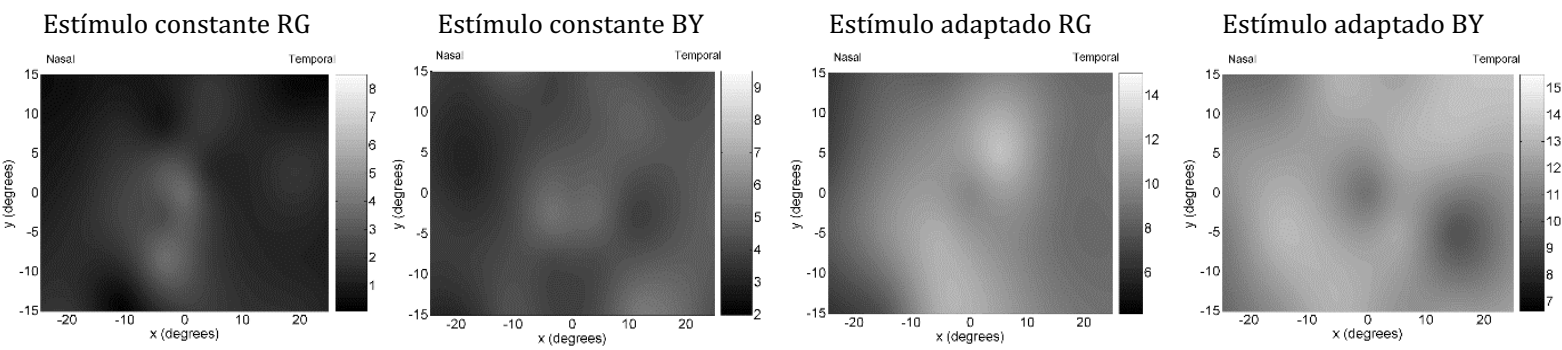

Fig. 8. Sensibilidad promedio (escala de gris) para el estímulo: constante RG y BY; adaptado a la excentricidad RG y BY.

Los resultados, que se presentan en la Figura 8, mostraron que para el mecanismo RG el aumento del tamaño del estímulo compensa la pérdida de sensibilidad, pero los resultados no son tan evidentes en el caso del mecanismo BY. El cambio de paradigma, además, mejoró la concordancia en las localizaciones periféricas de la sensibilidad medida en dos mediciones diferentes realizadas por un mismo observador. 


\section{Conclusiones}

El trabajo presente muestra los resultados recogidos en la tesis doctoral de García-Domene [33]. Se han llevado a cabo los pasos necesarios para crear un nuevo campímetro de análisis de los canales visuales cromáticos por proyección, para estímulos de tamaño constante y tamaño variable adaptado a la excentricidad, y se ha realizado un estudio previo de su fiabilidad.

Se ha creado un protocolo de calibrado y caracterización para cualquier dispositivo, con el que, a partir de las medidas en pocas localizaciones de la proyección, un modelo matemático y un proceso de interpolación podemos caracterizar cualquier píxel. Con esta preparación del proyector, el dispositivo puede utilizarse para múltiples tareas psicofísicas y optométricas, como podrían ser medidas de CSF o terapia visual, gracias tanto a la buena resolución espacial como a la extensión de la proyección.

En particular se ha implementado en entorno Matlab ${ }^{\circledR}$ un programa para medir el umbral de sensibilidad cromática presentando estímulos que se ajustan a las características de las células de los mecanismos cromáticos del sistema visual humano al que hemos llamado Multicampímetro [34]. Este programa incluye un análisis de los resultados obtenidos con un paciente en comparación con nuestra base de datos de sujetos normales [35]. Para ello se utilizó una versión modificada del método de medida MOBS [29] que no ha sido utilizado todavía en la medida de campimetrías.

Se ha realizado un estudio de repetitividad en el cual se demuestra que las medidas con el Multicampímetro son repetibles. Y se ha realizado una base de datos de sujetos normales que sigue las propiedades de los campos visuales descritos en la literatura [32]. Además, se han realizado las primeras pruebas con estímulos adaptados a la excentricidad obteniendo resultados prometedores.

Las perspectivas de futuro de este dispositivo son:

1- Ampliar la base de datos al resto de edades.

2- Las medidas preliminares muestran una mejora de la repetitividad y de la sensibilidad en periferia con el estímulo de tamaño variable, por lo que nos disponemos a realizar una base de datos por edades de dicha campimetría para ambos canales cromáticos.

3- Realizar más medidas en pacientes patológicos o anómalos cromáticos ya diagnosticados con el fin de comprobar mejor la sensibilidad del dispositivo.

4- Implementar otro tipo de pruebas psicofísicas: CSF, estímulos en movimiento o pruebas de terapia visual.

Acknowledgements: Agradecemos a la Cátedra Alcon-Universitat de València su apoyo económico para las becas recibidas de formación predoctoral. 